A. Murase and T. Sugano

Nagoya Math. J.

Vol. 132 (1993), 91-114

\title{
ZETA FUNCTIONS OF PREHOMOGENEOUS AFFINE SPACES
}

\author{
ATSUSHI MURASE AND TAKASHI SUGANO
}

\section{§0. Introduction}

Let $\rho$ be an algebraic homomorphism of a linear algebraic group $G$ into the affine transformation group $\operatorname{Aff}(V)$ of a finite dimensional vector space $V$. We say that a triplet $(G, V, \rho)$ is a prehomogeneous affine space, if there exists a proper algebraic subset $S$ of $V$ such that $V-S$ is a single $\rho(G)$-orbit. In particular, $(G, V, \rho)$ is a usual prehomogeneous vector space (PV, briefly) in the case where $\rho(G) \subset G L(V)$ (cf. [5], [7]). In the preceding paper [2], we defined zeta functions associated with certain prehomogeneous affine spaces and proved their analytic continuation and functional equations.

In the case of the PV's, M. Sato and Shintani [8] and F. Sato [3] established the theory of zeta functions associated with regular PV's (for the definition of a regular PV, see [5, Ch. 1, §1] or [7, §4, Definition 7]). Thus it is desirable to expand a similar theory for general prehomogeneous affine spaces. However there seems to be no appropriate definition of the dual of a prehomogeneous affine space and this causes a serious difficulty in studying zeta functions in the framework of prehomogeneous affine spaces.

In the present paper, we introduce the notion of an affine datum $\mathbf{D}=(G, V$, $\rho, \alpha)$ and its dual $\mathbf{D}^{*}$, where $\rho: G \rightarrow \operatorname{Aff}(V)$ is an algebraic homomorphism and $\alpha: V \times G \rightarrow \mathbf{G}_{a}$ is an affine 1-cocycle with respect to $\rho$. We say that $\mathbf{D}$ is a prehomogeneous affine datum (briefly, PAD) if $(G, V, \rho)$ is prehomogeneous. As in the case of the PV's, the dual of a PAD is not necessarily prehomogeneous and we are led to introduce the notion of a refular PAD (for definition, see §2). In fact, we show that the dual of a regular PAD is also prehomogeneous and regular (Proposition 2.4). The object of the paper is to define zeta functions associated with regular PAD's and prove their analytic continuation and functional equations

Received August 25, 1989.

Revised January 14, 1992. 
under certain mild assumptions.

We now explain a brief account of each section. In the first section, we state several elementary properties of a PAD without proofs since they are to be shown by the standard arguments in the theory of PV's. In $\S 2$, regular PAD's are defined and their fundamental properties are proved. The next two sections are devoted to the study of $a$-functions and $b$-functions of a regular PAD. The proofs are done by the arguments used in [5] with a slight modification (see also [6]). In $\S 5$, we consider (modified) complex powers of relative invariants of a regular PAD and study their Fourier transforms. In $\S 6$, we introduce zeta functions associated with a regular PAD and prove their functional equations by using the Poisson summation formula together with the results of $\S 5$. In the last $\S 7$, we explain several example of PAD's, one of which is closely related to the classical Hurwitz-Lerch zeta functions.

The authors would like to express their gratitude to Prof. F. Sato and Dr. H. Ochiai for their valuable suggestions, by which the authors are able to improve Proposition 5.1. They are also grateful to the referee for helpful advices.

Notation. As usual, we denote by $\mathbf{Z}, \mathbf{Q}, \mathbf{R}$ and $\mathbf{C}$ the ring of rational integers, the rational number field, the real number field and the complex number field, respectively. We put $\mathbf{e}[x]=\exp (2 \pi i x)$ for $x \in \mathbf{C}$. For a finite dimensional vector space $V$ over $\mathbf{R}, \oiint(V)$ stands for the space of rapidly decreasing smooth functions on $V$. Denote by $V^{*}$ the dual of $V$. The contragredient $A^{\sim} \in G L\left(V^{*}\right)$ of $A \in G L(V)$ is defined to be $\left\langle A x, A^{\sim} x^{*}\right\rangle=\left\langle x, x^{*}\right\rangle\left(x \in V, x^{*} \in V^{*}\right)$, where $\langle$,$\rangle is the natural pairing of V$ and $V^{*}$

\section{§1. Prehomogeneous affine datum}

Let $G$ be a connected linear algebraic group and $V$ a finite dimensional vector space with a right $G$-action $\rho$, all defined over $\boldsymbol{C}$. Assume that $\rho$ is an affine action (that is to say, $\rho$ defines an algebraic homomorphism of $G$ into the group $\operatorname{Aff}(V)$ of affine transformations of $V$ ). A regular rational function $\alpha$ on $V \times G$ is called an affine 1-cocycle with respect to $\rho$ if $\alpha$ satisfies the cocycle condition

$$
\alpha\left(x, g g^{\prime}\right)=\alpha\left(x \rho(g), g^{\prime}\right)+\alpha(x, g) \quad\left(x \in V, g, g^{\prime} \in G\right)
$$

and if the mapping $x \rightarrow \alpha(x, g)-\alpha(0, g)$ is linear on $V$ for every $g \in G$. We call a quartet $\mathbf{D}=(G, V, \rho, \alpha)$ an affine datum if $\alpha$ is an affine cocycle with respect to $\rho$.

Denote by $\left\langle x, x^{*}\right\rangle$ the natural pairing of $V$ and its dual $V^{*}$. The dual $\mathbf{D}^{*}$ of 
an affine datum $\mathbf{D}=(G, V, \rho, \alpha)$ is defined to be an affine datum $\left(G, V^{*}, \rho^{*}\right.$, $\left.\alpha^{*}\right)$ which satisfies

$$
\left\langle x \rho(g), x^{*}\right\rangle+\alpha(x, g)=\left\langle x, x^{*} \rho^{*}\left(g^{-1}\right)\right\rangle+\alpha^{*}\left(x^{*}, g^{-1}\right)
$$

for any $x \in V, x^{*} \in V^{*}$ and $g \in G$. It is easy to see that the dual $\mathbf{D}^{*}$ always exists and is uniquely determined by the condition (1.2). To describe $\mathbf{D}^{*}$ in an explicit manner, we define $a(g) \in G L(V), b(g) \in V$ and $c^{*}(g) \in V^{*}$ to be

$$
\begin{gathered}
x \rho(g)=a(g)(x)+b(g) \\
\alpha(x, g)=\left\langle x, c^{*}(g)\right\rangle+\alpha(0, g) .
\end{gathered}
$$

Then $\rho^{*}$ and $\alpha^{*}$ are given by

$$
\begin{gathered}
x^{*} \rho^{*}(g)=a(g) \sim x^{*}+c^{*}\left(g^{-1}\right) \\
\alpha^{*}\left(x^{*}, g\right)=\left\langle b\left(g^{-1}\right), x^{*}\right\rangle+\alpha\left(0, g^{-1}\right)
\end{gathered}
$$

for $x^{*} \in V^{*}$ and $g \in G$. Note that the dual of $\mathbf{D}^{*}$ is $\mathbf{D}$.

An affine datum $\mathbf{D}=(G, V, \rho, \alpha)$ is called a prehomogeneous affine datum (briefly a PAD) if there exists a proper algebraic subset $S$ of $V$ such that $V-S$ is a single $G$-orbit under $\rho$. We call $S$ the singular set of $\mathbf{D}$. Note that the dual of a PAD is not necessarily prehomogeneous.

Let $\mathbf{D}=(G, V, \rho, \alpha)$ be a $\mathrm{PAD}$. Let $X(G)$ be the group of rational characters of $G$. A non-zero rational function $P$ on $V$ is called a relative invariant of $\mathbf{D}$ corresponding to $\chi \in X(G)$ if $\mathrm{P}(x \rho(g))=\chi(g) \mathrm{P}(x)(x \in V, g \in G)$. As in the case of PV's, a relative invariant is uniquely determined by the corresponding character up to a constant multiple and any prime divisor of a relative invariant is also a relative invariant. Note that relative invariants are not necessarily homogeneous in our case (compare with [7]; see $\$ 7$ for examples of nonhomogeneous relative invariants).

For any subfield $K$ of $\mathbf{C}$, an affine datum $\mathbf{D}=(G, V, \rho, \alpha)$ is said to be $d e$ fined over $K$ if $G$ and $V$ admit $K$-structures such that $\rho$ and $\alpha$ are defined over $K$. It is obvious that the dual $\mathbf{D}^{*}$ of $\mathbf{D}$ is defined over $K$ if so is $\mathbf{D}$. In the remaining part of this section, we assume that $\mathbf{D}=(G, V, \rho, \alpha)$ is a $\mathrm{PAD}$ defined over a fixed subfield $K$ of $\mathbf{C}$. Denote by $G_{1}$ the normal closed subgroup of $G$ generated by the commutator subgroup $[G, G]$ of $G$ and the stabilizer $G(x)=\{g \in G \mid$ $x \rho(g)=x\}$ for a generic point $x \in V-S$. The group $G_{1}$ does not depend on the choice of $x$. Put $X_{\rho}(G)=\left\{\chi \in X(G) \mid \chi\right.$ is trivial on $\left.G_{1}\right\}$. It is shown that $X_{\rho}(G)$ coincides with the group of rational characters of $G$ corresponding to relative invariants of $\mathbf{D}$ (cf. [7, $\S 4$ Prop. 19]). Denote by $X_{\rho}(G)_{K}$ the subgroup of 
$X_{\rho}(G)$ consisting of rational characters in $X_{\rho}(G)$ defined over $K$. The next two lemmas are proved in the same manner as Lemma 1.1 and Lemma 1.2 of [3].

Lemma 1.1. Let $S$ be the singular set of $\mathbf{D}$ and $S^{\prime}$ the union of the irreducible components of $S$ of codimension one. Then both of $S$ and $S^{\prime}$ are defined over $K$.

Lemma 1.2. (i) There exists a finite Galois extension $L$ of $K$ such that every relative invariant of $\mathbf{D}$ is expressed as a product of a complex number and a rational function with coefficients in $L$.

(ii) Let $P(x)$ be a relative invariant of $\mathbf{D}$ corresponding to $\chi \in X_{\rho}(G)$. Then $P(x)$ is expressed as a product of a complex number and a rational function with coefficients in $K$ if and only if $\chi \in X_{\rho}(G)_{K}$.

Let $S_{1}, \ldots, S_{n}$ be the $K$-irreducible components of $S$ of codimension one and $P_{1}, \ldots, P_{n}$ be $K$-irreducible polynomials that define $S_{1}, \ldots, S_{n}$ respectively. The following results are proved in quite a similar manner as in the case of the PV's (see $[3, \S 1]$ ).

Lemma 1.3. The polynomials $P_{1}, \ldots, P_{n}$ are algebraically independent relative invariants corresponding to $\chi_{1}, \ldots, \chi_{n} \in X_{\rho}(G)_{K}$ respectively. Furthermore any relative invariant $P(x)$ with coefficients in $K$ is of the form $P(x)=c \cdot P_{1}(x)^{m_{1}} \cdots P_{n}(x)^{m_{n}}$ $\left(c \in K, m_{1}, \ldots, m_{n} \in \mathbf{Z}\right)$.

Lemma 1.4. The group $X_{\rho}(G)_{K}$ is a free $\mathbf{Z}$-module of rank $n$ generated by $\chi_{1}, \ldots, \chi_{n}$.

\section{§2. $K$-regular PAD}

In this section we let $\mathbf{D}$ be a PAD defined over a fixed subfield $K$ of $\mathbf{C}$. The next lemma is easily verified.

Lemma 2.1. The following conditions are equivalent:

(i ) $\alpha\left(x_{0}, G\left(x_{0}\right)\right)=0$ for some $x_{0} \in V-S$.

(ii) $\alpha(x, G(x))=0$ for any $x \in V-S$.

(iii) There exists a regular rational function $\beta$ on $V-S$ that is defined over $K$ and satisfies

$$
\alpha(x, g)=\beta(x \rho(g))-\beta(x) \quad(x \in V-S, g \in G) .
$$


(Recall that $G(x)$ is the stabilizer subgroup of $x$ in $G$.)

From now on, we always assume that $\mathbf{D}$ satisfies the above equivalent conditions and fix a function $\beta$ satisfying (2.1) once and for all. Let $\mathfrak{g}, g_{1}$ and $\mathfrak{g}(x)(x \in$ $V-S$ ) be the Lie algebras of $G, G_{1}$ and $G(x)$, respectively. For $x \in V$ and $A \in$ g, put

$$
\begin{gathered}
x d \rho(A)=\left.\frac{d}{d t}\{x \rho(\exp (t A))\}\right|_{t=0} \in V \\
d \alpha(x, A)=\left.\frac{d}{d t}\{\alpha(x, \exp (t A))\}\right|_{t=0} \in \mathbf{C} .
\end{gathered}
$$

Our assumption implies

$$
d \alpha(x, \mathfrak{g}(x))=0 \quad(x \in V-S) .
$$

The next lemma follows from a straightforward calculation.

Lemma 2.2. For $x \in V, g \in G$ and $A \in \mathfrak{g}$, we have

$$
x d \rho(\operatorname{Ad}(g) A)=x \rho(g) d \rho(A) \rho\left(g^{-1}\right)-0 \cdot \rho\left(g^{-1}\right),
$$

$$
d \alpha(x, \operatorname{Ad}(g) A)=\alpha\left(x \rho(g) d \rho(A), g^{-1}\right)+d \alpha(x \rho(g), A)-\alpha\left(0, g^{-1}\right)
$$

where Ad stands for the adjoint representation of $G$ on $\mathrm{g}$.

A rational mapping $\phi$ of a $G$-stable Zariski open subset $V^{\prime}$ of $V$ into $V^{*}$ is said to be $G$-equivariant if $\phi(x \rho(g))=\phi(x) \rho^{*}(g) \quad\left(x \in V^{\prime}, g \in G\right)$. We denote by $\mathrm{g}^{*}$ the dual of $\mathrm{g}$.

Proposition 2.3. For $\omega \in \mathrm{g}^{*}$, the following two assertions are equivalent.

(i) There exists a unique $G$-equivariant rational mapping $\phi_{\omega}: V-S \rightarrow V^{*}$ satisfying

$$
\left\langle x d \rho(A), \phi_{\omega}(x)\right\rangle+d \alpha(x, A)=\omega(A) \quad(x \in V-S, A \in \mathrm{g}) .
$$

(ii) $\omega$ vanishes on $g_{1}$.

Proof. (i) $\Rightarrow$ (ii): Take an $x \in V-S$. Since $g(x)=\{A \in g \mid x d \rho(A)=0\}$, (2.7) and (2.4) imply that $\omega(\mathfrak{g}(x))=0$. It remains to prove that $\omega([\mathrm{g}, \mathrm{g}])=0$, since $g_{1}$ is generated by $g(x)$ and $[g, g]$. Chaging $x$ (resp. A) into $x \rho\left(g^{-1}\right)$ (resp. $\operatorname{Ad}(g) A)$ in $(2.7)$ and applying Lemma 2.2 , we have

$$
\omega(\operatorname{Ad}(g) A)=\left\langle x d \rho(A) \rho\left(g^{-1}\right)-0 \cdot \rho\left(g^{-1}\right), \phi_{\omega}(x) \rho^{*}\left(g^{-1}\right)\right\rangle
$$




$$
+\alpha\left(x d \rho(A), g^{-1}\right)+d \alpha(x, A)-\alpha\left(0, g^{-1}\right) .
$$

It follows from (1.3), (1.4) and (1.5) that

$$
\begin{aligned}
& \left\langle x d \rho(A) \rho\left(g^{-1}\right)-0 \cdot \rho\left(g^{-1}\right), \phi_{\omega}(x) \rho^{*}\left(g^{-1}\right)\right\rangle \\
& =\left\langle x d \rho(A), \phi_{\omega}(x)\right\rangle+\alpha\left(x d \rho(A) \rho\left(g^{-1}\right), g\right)-\alpha\left(0 \cdot \rho\left(g^{-1}\right), g\right) .
\end{aligned}
$$

Since $\alpha\left(x d \rho(A), g^{-1}\right)+\alpha\left(x d \rho(A) \rho\left(g^{-1}\right), g\right)-\alpha\left(0, g^{-1}\right)-\alpha\left(0 \cdot \rho\left(g^{-1}\right), g\right)=0$ by the cocycle condition (1.1), we obtain $\omega(\operatorname{Ad}(g) A)=\omega(A)$. This implies that $\omega([\mathrm{g}, \mathrm{g}])=0$.

(ii) $\Rightarrow$ (i): Let $x \in V-S$. Since $\omega(A)-\mathrm{d} \alpha(x, A)=0$ for any $A \in \mathrm{g}(x)$ and since $A \rightarrow x d \rho(A)$ induces a linear isomorphism of $\mathrm{g} / \mathrm{g}(x)$ onto $V$, there uniquely exists $\phi_{\omega}(x) \in V^{*}$ with $\left\langle x d \rho(A), \phi_{\omega}(x)\right\rangle=\omega(A)-d \alpha(x, A)$ for $A \in \mathfrak{g}$. Then $x \rightarrow \phi_{\omega}(x)$ defines a regular rational mapping of $V-S$ to $V^{*}$. We now prove the $G$-equivariance of $\phi_{\omega}$. Since $\omega(\operatorname{Ad}(g) A)=\omega(A)(A \in g, g \in G)$, we have

$$
\begin{aligned}
\left\langle x \rho(g) d \rho(A), \phi_{\omega}(x \rho(g))\right\rangle+d \alpha(x \rho(g), A) & \\
= & \left\langle x d \rho(\operatorname{Ad}(g) A), \phi_{\omega}(x)\right\rangle+d \alpha(x, \operatorname{Ad}(g) A) .
\end{aligned}
$$

Applying Lemma 2.2, we obtain

$$
\begin{aligned}
& \left\langle x \rho(g) d \rho(A), \phi_{\omega}(x \rho(g))\right\rangle \\
& =\left\langle x \rho(g) d \rho(A) \rho\left(g^{-1}\right)-0 \cdot \rho\left(g^{-1}\right), \phi_{\omega}(x)\right\rangle+\alpha\left(x \rho(g) d \rho(A), g^{-1}\right)-\alpha\left(0, g^{-1}\right) \\
& =\left\langle x \rho(g) d \rho(A) \rho\left(g^{-1}\right), \phi_{\omega}(x)\right\rangle+\alpha\left(x \rho(g) d \rho(A), g^{-1}\right)-\alpha^{*}\left(\phi_{\omega}(x), g\right) .
\end{aligned}
$$

(Note that $\left\langle 0 \cdot \rho\left(g^{-1}\right), \phi_{\omega}(x)\right\rangle+\alpha\left(0, g^{-1}\right)=\alpha^{*}\left(\phi_{\omega}(x), g\right)$.) In view of the relation (1.2), we have $\left\langle x \rho(g) d \rho(A), \phi_{\omega}(x \rho(g))\right\rangle=\left\langle x \rho(g) d \rho(A), \phi_{\omega}(x) \rho^{*}(g)\right\rangle$ for $A \in \mathrm{g}$, which proves the assertion. q.e.d.

A PAD $\mathbf{D}$ is said to be quasi-regular if $\mathbf{D}$ satisfies the conditions in Lemma 2.1 and if there exists $\omega \in X_{1}^{*}=\left\{\omega \in \mathrm{g}^{*} \mid \omega\right.$ vanishes on $\left.\mathrm{g}_{1}\right\}$ such that $\phi_{\omega}$ is dominant (that is, the image of $\phi_{\omega}$ is Zariski dense in $V^{*}$ ). In this case $\omega$ is said to be non-degenerate. Let $\chi_{1}, \ldots, \chi_{n}$ be the $K$-rational characters of $G$ defined by $P_{i}(x \rho(g))=\chi_{i}(g) P_{i}(x)$ as in $\S 1$. Since their infinitesimal characters $d \chi_{1}, \ldots, d \chi_{n}$ vanish on $\mathfrak{g}_{1}, X_{0}^{*}=\mathbf{C} d \chi_{1}+\cdots+\mathbf{C} d \chi_{n}$ is a subspace of $X_{1}^{*}$. A quasi-regular PAD defined over $K$ is said to be $K$-regular if there exists a non-degenerate element $\omega \in X_{0}^{*}$.

Proposition 2.4. If $\mathbf{D}$ is a quasi-regular (resp. $K$-regular) $P A D$, then its dual $\mathbf{D}^{*}$ is also a quasi-regular (resp. K-regular) PAD. Furthermore if $\omega$ is a nondegenerate element, then $\phi_{\omega}$ gives a one-to-one biregular rational mapping of $V-S$ 
onto $V^{*}-S^{*}\left(S^{*}\right.$ is the singular set of $\left.\mathbf{D}^{*}\right)$ and $G^{*}\left(\phi_{\omega}(x)\right)=\{g \in G \mid$ $\left.\phi_{\omega}(x) \rho^{*}(g)=\phi_{\omega}(x)\right\}$ coincides with $G(x)$ for any $x \in V-S$.

Proof. Let $\omega \in X_{1}^{*}$ (resp. $\omega \in X_{0}^{*}$ ) be a non-degenerate element. Then $\phi_{\omega}(V$ $-S)$ is a Zariski dense $G$-orbit under the action $\rho^{*}$. Hence the affine datum $\mathbf{D}^{*}=\left(G, V^{*}, \rho^{*}, \alpha^{*}\right)$ is prehomogeneous and $\phi_{\omega}(V-S)=V^{*}-S^{*}$ where $S^{*}$ is the singular set of $\mathbf{D}^{*}$. For $x \in V-S$, put $x^{*}=\phi_{\omega}(x)$ and $G^{*}\left(x^{*}\right)=\{g \in G$ $\left\{x^{*} \rho^{*}(g)=x^{*}\right\}$. Then we have $G(x) \subset G^{*}\left(x^{*}\right)$ and $\operatorname{dim} G^{*}\left(x^{*}\right)=\operatorname{dim} G-$ $\operatorname{dim} V=\operatorname{dim} G(x)$. This implies that $g_{1}$ coincides with the Lie algebra of $G_{1}^{*}$, the group generated by $G^{*}\left(x^{*}\right)$ and the commutator subgroup $[G, G]$. By Proposition 2.3 , there exists a rational mapping $\phi_{\omega}: V^{*}-S^{*} \rightarrow V$ satisfying $\phi_{\omega}\left(x^{*} \rho^{*}(g)\right)=$ $\phi_{\omega}\left(g^{*}\right) \rho(g)$ and $\left\langle\phi_{\omega}\left(x^{*}\right), x^{*} d \rho^{*}(A)\right\rangle+d \alpha^{*}\left(x^{*}, A\right)=-\omega(A)\left(x^{*} \in V^{*}-\right.$ $\left.S^{*}, g \in G, A \in \mathfrak{g}\right)$. Therefore we have

$$
\begin{aligned}
& \left\langle x, x^{*} d \rho^{*}(A)\right\rangle=\left\langle-x d \rho(A), \phi_{\omega}(x)\right\rangle-d \alpha(x, A)-d \alpha^{*}\left(x^{*}, A\right) \\
& =-\omega(A)-d \alpha^{*}\left(x^{*}, A\right)=\left\langle\phi_{\omega}\left(x^{*}\right), x^{*} d \rho^{*}(A)\right\rangle
\end{aligned}
$$

for any $A \in \mathrm{g}$. This implies $\phi_{\omega}\left(\phi_{\omega}(x)\right)=x$ for $x \in V-S$. A similar argument shows that $\phi_{\omega}{ }^{\circ} \phi_{\omega}$ is the identity mapping on $V^{*}-S^{*}$. Thus $\phi_{\omega}$ is a one-to-one biregular mapping of $V-S$ onto $V^{*}-S^{*}$ and $\phi_{\omega}$ is its inverse. The remaining part of the proposition follows from this fact.

q.e.d.

Corollary 2.5. Let $\mathbf{D}=(G, V, \rho, \alpha)$ be a quasi-regular $P A D$ defined over $K$ and $\mathbf{D}^{*}=\left(G, V^{*}, \rho^{*}, \alpha^{*}\right)$ its dual. Then

(i ) $G_{1}=G_{1}^{*}$.

(ii) The number $n^{*}$ of $K$-irreducible components of $S^{*}$ of codimension one is equal to that of $S$.

(iii) $X_{\rho}(G)_{K}=X_{\rho}(G)_{K}$.

Proof. These are easily deduced from Proposition 2.4 if we observe $n^{*}=$ $\operatorname{rank} X_{\rho}(G)_{K}$ and $X_{\rho} *(G)_{K}=X\left(G / G_{1}^{*}\right)_{K}$.

q.e.d.

Fix a basis of $V$ and let $\left(x_{1}, \ldots, x_{N}\right)$ be the coordinate of $x \in V(N=\operatorname{dim} V)$. From now on, coordinates of elements of $V^{*}$ are taken to be with respect to the dual basis of the above one. For a smooth function $f$ on $V$, we define $\operatorname{grad} f: V \rightarrow$ $V^{*}$ to be $\operatorname{grad} f(x)=\left(\frac{\partial f}{\partial x_{1}}, \ldots, \frac{\partial f}{\partial x_{N}}\right)$. It is easy to see that this definition does not depend upon the choice of a basis of $V$. If $f$ does not vanish on $V-S$, we put $\operatorname{grad} \log f(x)=f(x)^{-1} \operatorname{grad} f(x) \quad(x \in V-S)$. For $s=\left(s_{1}, \ldots, s_{n}\right) \in \mathbf{C}^{n}$, put 


$$
\omega_{s}=\sum_{1 \leq i \leq n}\left(-\frac{s_{i}}{2 \pi i}\right) d \chi_{i} \in X_{0}^{*}
$$

Lemma 2.6. We have

$$
\phi_{\omega_{s}}(x)=-\frac{1}{2 \pi i} \sum_{1 \leq i \leq n} s_{i} \cdot \operatorname{grad} \log P_{i}(x)-\operatorname{grad} \beta(x) .
$$

Proof. Differentiating the equalities $\alpha(x, \exp (t A))=\beta(x \rho(\exp (t A)))-$ $\beta(x)$ and $P_{i}(x \rho(\exp (t A)))=\chi_{i}(\exp (t A)) \cdot P_{i}(x) \quad(x \in V-S, A \in g)$ at $t=0$, we obtain $d \alpha(x, A)=\langle x d \rho(A), \operatorname{grad} \beta(x)\rangle$ and $d \chi_{i}(A)=\langle x d \rho(A)$, grad log $\left.P_{i}(x)\right\rangle$. These prove the assertion of the lemma.

q.e.d.

For $x \in V-S$, we define the differential mapping $d \phi_{\omega}(x): V \rightarrow V^{*}$ of $\phi_{\omega}$ to be $d \phi_{\omega}(x)(y)=\left.\frac{d}{d t}\left(\phi_{\omega}(x+t y)\right)\right|_{t=0}$ for $y \in V$. A straightforward calculation shows $d \phi_{\omega}(x \rho(g))(a(g) y)=a(g)^{\sim} d \phi_{\omega}(x)(y)(x \in V-S, y \in V, g \in G)$, which implies that $\operatorname{det}\left(d \phi_{\omega}(x \rho(g))\right)=\chi_{0}(g)^{-1} \operatorname{det}\left(d \phi_{\omega}(x)\right) \quad(x \in V-S, g \in G)$ with

$$
\chi_{0}(g)=(\operatorname{det} a(g))^{2} .
$$

Thus we have proved

Lemma 2.7. If $\mathbf{D}=(G, V, \rho, \alpha)$ is defined over $K$ and quasi-regular, then $\chi_{0} \in X_{\rho}(G)_{K}$.

Lemma 2.8. Let $\mathbf{D}$ be a $P A D$ and $\omega \in X_{1}^{*}$. Then

$$
\frac{\partial \phi_{\omega}(x)_{j}}{\partial x_{i}}=\frac{\partial \phi_{\omega}(x)_{i}}{\partial x_{j}} \quad(1 \leq i, j \leq N)
$$

where $\phi_{\omega}(x)=\left(\phi_{\omega}(x)_{i}\right)_{1 \leq i \leq N} \in V^{*}$.

Proof. For $x=\left(x_{1}, \ldots, x_{N}\right) \in V, A \in \mathfrak{g}$ and $1 \leq i \leq N$, we put

$$
(x \cdot d \rho(A))_{i}=\sum_{1 \leq j \leq N} x_{j} a_{j i}(A)+b_{i}(A), d \alpha(x, A)=\sum_{1 \leq i \leq N} \alpha_{i}(A) x_{i}+d \alpha(0, A)
$$

$\left(a_{j i}(A), b_{i}(A), \alpha_{i}(A) \in \mathbf{C}\right)$. Then it is easy to see that $\left(x^{*} d \rho^{*}(A)\right)_{i}=$ $-\sum_{1 \leq j \leq N} x_{j}^{*} a_{i j}(A)-\alpha_{i}(A)$ for $x^{*}=\left(x_{1}^{*}, \ldots, x_{N}^{*}\right) \in V^{*}$. It follows from (2.7) that 


$$
\sum_{1 \leq i, j \leq N}\left(x_{j} a_{j i}(A)+b_{i}(A)\right) \phi_{\omega}(x)_{i}+\sum_{1 \leq i \leq N} x_{i} \alpha_{i}(A)+\alpha(0, A)=\omega(A) .
$$

Differentiating the above formula in $x_{k}$, we obtain

$$
\sum_{1 \leq i \leq N} a_{k i}(A) \phi_{\omega}(x)_{i}+\sum_{1 \leq i, j \leq N}\left(x_{j} a_{j i}(A)+b_{i}(A)\right) \frac{\partial \phi_{\omega}(x)_{i}}{\partial x_{k}}+\alpha_{k}(A)=0 .
$$

On the other hand, the $G$-equivariance of $\phi_{\omega}$ implies

$$
\phi_{\omega}(x \rho(\exp (t A)))_{k}=\left(\phi_{\omega}(x) \rho^{*}(\exp (t A))\right)_{k} .
$$

Differentiating (2.12) at $t=0$, we get

$$
\begin{aligned}
\sum_{1 \leq i, j \leq N} \frac{\partial \phi_{\omega}(x)_{k}}{\partial x_{i}} & \left(x_{j} a_{j i}(A)+b_{i}(A)\right) \\
= & -\sum_{1 \leq i \leq N}+\alpha_{k i}(A) \phi_{\omega}(x)_{i}-\alpha_{k}(A) \quad(1 \leq k \leq N)
\end{aligned}
$$

Comparing (2.11) and (2.13), we prove the lemma.

q.e.d.

\section{§3. The $a$-functions}

Let $\mathbf{D}=(G, V, \rho, \alpha)$ be a $K$-regular $\mathrm{PAD}$ and $\mathbf{D}^{*}=\left(G, V^{*}, \rho^{*}, \alpha^{*}\right)$ its dual. Let $S_{1}, \ldots, S_{n}$ (resp. $S_{1}^{*}, \ldots, S_{n}^{*}$ ) be the $K$-irreducible components of the singular set $S$ (resp. $S^{*}$ ) of $\mathbf{D}$ (resp. $\mathbf{D}^{*}$ ) of codimension one and $P_{1}, \ldots, P_{n}$ (resp. $\left.P_{1}^{*}, \ldots, P_{n}^{*}\right)$ be their defining equations in $K[V]$ (resp. $K\left[V^{*}\right]$ ). Let $\chi_{i}$ (resp. $\chi_{i}^{*}$ ) be the corresponding character to the relative invariant $P_{i}$ (resp. $P_{i}^{*}$ ) of $\mathbf{D}$ (resp. $\mathbf{D}^{*}$ ). By Lemma 1.4 and Corollary 2.5, we see that the group generated by $\chi_{i}(1 \leq i \leq n)$ coincides with the one generated by $\chi_{i}^{*}(1 \leq i \leq n)$. This implies

$$
\chi_{i}=\prod_{1 \leq j \leq n} \chi_{j}^{* u_{i j}} \quad(1 \leq i \leq n)
$$

with $U=\left(u_{i j}\right) \in G L_{n}(\mathbf{Z})$. Take $K$-rational functions $\beta$ and $\beta^{*}$ on $V-S$ and $V^{*}-S^{*}$ so that $\alpha(x, g)=\beta(x \rho(g))-\beta(x) \quad(x \in V-S, g \in G)$ and that $\alpha^{*}\left(x^{*}, g\right)=\beta^{*}\left(x^{*} \rho^{*}(g)\right)-\beta^{*}\left(x^{*}\right)\left(x^{*} \in V^{*}-S^{*}, g \in G\right)$, respectively.

For $s=\left(s_{1}, \ldots, s_{n}\right) \in \mathbf{C}^{n}$, we define rational mapping $\phi_{s}: V-S \rightarrow V^{*}$ and $\phi_{s}: V^{*}-S^{*} \rightarrow V$ as follows:

$$
\begin{gathered}
\phi_{s}(x)=\phi_{\omega_{s}}(x)=-\frac{1}{2 \pi i} \sum_{1 \leq i \leq n} s_{i} \cdot \operatorname{grad} \log P_{i}(x)-\operatorname{grad} \beta(x) \\
\phi_{s}\left(x^{*}\right)=\phi_{\omega_{s}}\left(x^{*}\right)=\frac{1}{2 \pi i} \sum_{1 \leq i \leq n} s_{i}^{\prime} \cdot \operatorname{grad} \log P_{i}^{*}\left(x^{*}\right)-\operatorname{grad} \beta^{*}\left(x^{*}\right)
\end{gathered}
$$


where $s^{\prime}=\left(s_{1}^{\prime}, \ldots, s_{n}^{\prime}\right)=s U$ (cf. proof of Proposition 2.4 and (2.8)). The following two lemmas are easily verified.

LEMMA 3.1. We have

$$
\begin{array}{r}
\phi_{s}(x \rho(g))=\phi_{s}(x) \rho^{*}(g), \phi_{s}\left(x^{*} \rho^{*}(g)\right)=\phi_{s}\left(x^{*}\right) \rho(g) \\
\left(x \in V-S, x^{*} \in V^{*}-S^{*}, g \in G\right) .
\end{array}
$$

Lemma 3.2. The set $\Omega=\left\{s \in \mathbf{C}^{n} \mid \phi_{s}\right.$ is dominant $\}$ is Zariski dense in $\mathbf{C}^{n}$. If $s \in \Omega$ then $\phi_{s}$ is dominant and we have $\phi_{s}{ }^{\circ} \phi_{s}=\mathrm{id}_{V^{*}-S^{*}}, \phi_{s}^{\circ} \phi_{s}=\mathrm{id}_{V-s}$.

For simplicity, we write $X_{K}$ for $X_{\rho}(G)_{K}=X_{\rho^{*}}(G)_{K}$. For $\chi \in X_{K}$, we define $d(\chi)=\left(d(\chi)_{1}, \ldots, d(\chi)_{n}\right), d^{*}(\chi)=\left(d^{*}(\chi)_{1}, \ldots, d^{*}(\chi)_{n}\right) \in \mathbf{Z}^{n}$ to be

$$
\chi=\prod_{1 \leq i \leq n} \chi_{i}^{d(\chi)_{i}}=\prod_{1 \leq i \leq n} \chi_{i}^{* d^{*}(\chi)_{i}}
$$

By $(3,1)$, we have $d^{*}(\chi)=d(\chi) U$. Put $P^{\chi}=\prod_{1 \leq i \leq n} P_{i}^{d(\chi)_{i}}$ and $P^{* \chi}=$ $\Pi_{1 \leq i \leq n}\left(P_{i}^{*}\right)^{d^{*}(\chi)_{i}}$, which are relative invariants of $\mathbf{D}$ and $\mathbf{D}^{*}$ respectively, both corresponding to $\chi$.

For $s \in \Omega$, the function $x \rightarrow P^{* \chi}\left(\phi_{s}(x)\right) \cdot P^{\chi^{-1}}(x)$ on $V-S$ is $\rho(G)$-invariant and hence constant on $V-S$. We denote its value by $a_{\chi}(s)$ and call this rational function on $\mathbf{C}^{n}$ the $\boldsymbol{a}$-function of $\mathbf{D}$ corresponding to $\chi$. The next lemma is deduced from the definition of $a_{\chi}(s)$.

LEMma 3.3. We have $a_{\chi \chi^{\prime}}(s)=a_{\chi}(s) \cdot a_{\chi^{\prime}}(s)$ for $\chi, \chi^{\prime} \in X_{K}$.

Lemma 3.4. If either $P^{* \chi}$ or $p^{\chi^{-1}}$ is a polynomial, then $a_{\chi}(s)$ is a polynomial of $s \in \mathbf{C}^{n}$.

Proof. This immediately follows from the equality $a_{\chi}(s)=P^{* x}\left(\phi_{s}(x)\right)$. $P^{x^{-1}}(x)=P_{x}^{*}\left(x^{*}\right) \cdot P^{x^{-1}}\left(\phi_{s}\left(x^{*}\right)\right)$ for $x \in V-S$ and $x^{*} \in V^{*}-S^{*}$.

q.e.d.

For $s \in \Omega$, let us consider the function on $V-S$ given by

$$
x \rightarrow\left\langle x, \phi_{s}(x)\right\rangle+\beta(x)+\beta^{*}\left(\phi_{s}(x)\right) .
$$

This function is invariant under $\rho(G)$ and hence constant on $V-S$. If we write $F(s)$ for its value, then $s \rightarrow F(s)$ defines a rational function on $\mathbf{C}^{n}$. In the remaining part of the paper, we always assume the following condition: 
(3.6) $F(s)$ is a non-constant polynomial of $s$.

Remark. Since $F(s)=\left\langle x^{*}, \phi_{s}\left(x^{*}\right)\right\rangle+\beta\left(\phi_{s}\left(x^{*}\right)\right)+\beta^{*}\left(x^{*}\right)$ for any $x^{*} \in$ $V^{*}-S^{*}$, the assumption is satisfied if either $\alpha$ or $\alpha^{*}$ is zero.

Proposition 3.5. Under the assumption (3.6), there exist distinct linear forms $e_{1}, \ldots, e_{m}$ on $\mathbf{C}^{n}$, natural numbers $M_{1}, \ldots, M_{m}$ and $c \in \operatorname{Hom}\left(X_{K}, \mathbf{C}^{\times}\right)$satisfying the following conditions:

( i ) $a_{\chi}(s)=c(\chi) \prod_{1 \leq j \leq m}\left(e_{j}(s)\right)^{-M_{j} e_{j}(d(x))}$

(ii) All the coefficients of $e_{j}$ are non-negative integers and $e_{j}\left(\mathbf{Z}^{n}\right)=\mathbf{Z}(1 \leq j \leq m)$.

Proof. Let $g_{1}(s), \ldots, g_{m}(s)$ be the distinct prime divisors of $a_{\chi_{1}}(s)^{-1}, \ldots$, $a_{x_{n}}(s)^{-1}$. By Lemma 3.3, there uniquely exist $\varepsilon_{1}, \ldots, \varepsilon_{m} \in \operatorname{Hom}\left(X_{K}, \mathbf{Z}\right)$ such that $a_{\chi}(s)=c_{\chi}^{\prime} \Pi_{1 \leq j \leq m} g_{j}(s)^{\varepsilon_{j}(\chi)}$ for $\chi \in X_{K}$, where $c_{\chi}^{\prime}$ is a constant depending only on $\chi$. Define linear forms $e_{1}^{\prime}, \ldots, e_{m}^{\prime}$ on $\mathbf{C}^{n}$ by $e_{j}^{\prime}(s)=\Pi_{1 \leq i \leq n} s_{i} \cdot \varepsilon_{j}\left(\chi_{i}\right)\left(s=\left(s_{1}, \ldots\right.\right.$, $\left.s_{n}\right) \in \mathbf{C}^{n}$ ). Since $e_{j}^{\prime}(d(\chi))=\varepsilon_{j}(\chi)$, we have $e_{j}^{\prime}\left(\mathbf{Z}^{n}\right) \subset \mathbf{Z}$ and hence we can find linear forms $e_{1}, \ldots, e_{m}$ and natural numbers $M_{1}, \ldots, M_{m}$ such that $e_{j}^{\prime}=-M_{j} e_{j}$ and $e_{j}\left(\mathbf{Z}^{n}\right)=\mathbf{Z}$. Since $\varepsilon_{j}\left(\chi_{j}\right) \leq 0(1 \leq i \leq n, 1 \leq j \leq m)$, we see that all the coefficients of $e_{j}$ are non-negative integers. Thus we have

$$
P^{* x}\left(\phi_{s}(x)\right) \cdot P^{x^{-1}}(x)=c_{\chi}^{\prime} \prod_{1 \leq j \leq m} g_{j}(s)^{-M_{j} e_{j}(d(x))} \quad((x, s) \in(V-S) \times \Omega) .
$$

Recall that $\operatorname{grad} \log P^{x^{-1}}(x)=2 \pi i\left(\phi_{d(x)}(x)+\operatorname{grad} \beta(x)\right)$ and $\operatorname{grad} \log P^{* x}\left(x^{*}\right)$ $=2 \pi i\left(\phi_{d(x)}\left(x^{*}\right)+\operatorname{grad} \beta^{*}\left(x^{*}\right)\right)$. Taking the logarithmic derivatives of the both sides of (3.7), we obtain

$$
\begin{aligned}
& \phi_{d(\chi)}(y) d y+\operatorname{grad} \beta^{*}(y) d y+\phi_{d(x)}(x) d x+\operatorname{grad} \beta(x) d x \\
& =-\frac{1}{2 \pi i} \sum_{1 \leq j \leq m} M_{j} e_{j}(d(\chi)) \frac{d g_{j}(s)}{g_{j}(s)}
\end{aligned}
$$

for $\chi \in X_{K}$ and hence

$$
\begin{aligned}
& \phi_{s^{\prime}}(y) d y+\operatorname{grad} \beta^{*}(y) d y+\phi_{s^{\prime}}(x) d x+\operatorname{grad} \beta(x) d x \\
& =-\frac{1}{2 \pi i} \sum_{1 \leq j \leq m} M_{j} e_{j}\left(s^{\prime}\right) \frac{d g_{j}(s)}{g_{j}(s)}
\end{aligned}
$$

for any $s, s^{\prime} \in \mathbf{C}^{n}$, where we put $y=\phi_{s}(x)$. Putting $s^{\prime}=s$ in the above formula, we get 


$$
d F(s)=-\frac{1}{2 \pi i} \sum_{1 \leq i \leq m} M_{j} e_{j}(s) \frac{d g_{j}(s)}{g_{j}(s)} .
$$

By (3.8) and the assumption (3.6), $g_{j}(s)$ divides $e_{j}(s)$ and hence $g_{j}(s)$ is a constant multiple of $e_{j}(s)$. Thus the proof of the proposition is completed.

q.e.d.

COROLLARY 3.6. Under the assumption (3.6), we have $F(s)=-\frac{1}{2 \pi i}$ $\sum_{1 \leq i \leq n} \delta_{i} s_{i}+c$ with non-negative integers $\delta_{1}, \ldots, \delta_{n}$ and $c \in \mathbf{C}$. In fact, we have $\delta_{i}$ $=\sum_{1 \leq j \leq m} M_{j} e_{j i}(1 \leq i \leq n)$ where $e_{i j} \in \mathbf{Z}$ is defined to be $e_{j}(s)=\sum_{1 \leq i \leq n} e_{j i} s_{i}$ (note that $e_{j i} \geq 0$ ).

Proof. In the proof of Proposition 3.5, we have obtained the formula $d F(s)=\sum_{1 \leq j \leq m} M_{j} d e_{j}(s)$. Our assertion follows from this.

q.e.d.

Note that the constant term of $F(s)$ depends upon the choice of $\beta$ and $\beta^{*}$, though the linear term of $F(s)$ depends only upon $\mathbf{D}$. We say that $\left(\beta, \beta^{*}\right)$ is a normalized pair for $\left(\mathbf{D}, \mathbf{D}^{*}\right)$ if the constant term of $F(s)$ is zero. The following is an immediate consequence of Proposition 3.5 and Corollary 3.6.

COROLlary 3.7. If either $P^{\chi^{-1}}$ or $P^{* x}$ is a polynomial, then $a_{\chi}(s)$ is a homogeneous polynomial of $s \in \mathbf{C}^{n}$ of degree $-\sum_{1 \leq i \leq n} \delta_{i} d(\chi)_{i}$.

Remark. If $\mathbf{D}=(G, V, \rho, \alpha)$ is a $K$-regular $\mathrm{PV}$, that is, if $\operatorname{Im}(\rho) \subset$ $\mathrm{GL}(V)$ and $\alpha \equiv 0$, the assumption (3.6) is always satisfied and the integer $\delta_{i}$ is equal to the degree of $P_{i}$.

\section{\$4. The $b$-functions}

We keep the notation and the assumptions of $\S 3$ and furthermore assume that $K$ is a subfield of $\mathbf{R}$. For an integer $i(1 \leq i \leq N)$, let $D_{i}$ be the differential operator of the first order on $V_{\mathbf{R}}$ given by

$$
D_{i} f(x)=\frac{1}{2 \pi i} \frac{\partial f}{\partial x_{i}}(x)-\frac{\partial \beta}{\partial x_{i}}(x) \cdot f(x) \quad\left(x \in V_{\mathbf{R}}, f \in C^{\infty}\left(V_{\mathbf{R}}\right)\right) .
$$

Since the $D_{i}$ 's mutually commute, the differential operator

$$
R_{\chi}=P^{* \chi}\left(D_{1}, \ldots, D_{N}\right)
$$

on $V_{\mathbf{R}}$ is well-defined for $\chi \in X_{K}^{+}=\left\{\chi \in X_{K} \mid P^{* \chi}\right.$ is a polynomial $\}$. We write 
$R_{\chi}=P^{* \chi}\left(\frac{1}{2 \pi i} \operatorname{grad}-\operatorname{grad} \beta\right)$ symbolically.

Lemma 4.1. We have

$$
R_{\chi}\left(\mathbf{e}\left[\left\langle x, x^{*}\right\rangle+\beta(x)\right]\right)=P^{* x}\left(x^{*}\right) \cdot \mathbf{e}\left[\left\langle x, x^{*}\right\rangle+\beta(x)\right] .
$$

Proof. This follows from $D_{i}\left(\mathbf{e}\left[\left\langle x, x^{*}\right\rangle+\beta(x)\right]\right)=x_{i}^{*} \cdot \mathbf{e}\left[\left\langle x, x^{*}\right\rangle+\beta(x)\right]$.

Let $R_{\chi}^{\sim}$ be the adjoint operator of $R_{\chi}$ given by $R_{\chi}^{\sim}=P^{* x}\left(-\frac{1}{2 \pi i} \operatorname{grad}-\right.$ $\operatorname{grad} \beta)$. For $s=\left(s_{1}, \ldots, s_{n}\right) \in \mathbf{C}^{n}$, we put $P^{s}=P_{1}^{s_{1}} \cdots P_{n}^{s_{n}}$.

Lemma 4.2. For $\chi \in X_{K}^{+}$, there exists a polynomial $b_{\chi}(s)$ of $s \in \mathbf{C}^{n}$ such that

$$
R_{\chi}^{\sim}\left(P^{s}\right)=b_{\chi}(s) \cdot P^{s+d(\chi)} .
$$

Proof. For a function $\varphi$ on $V_{\mathbf{R}}$, we write $\varphi^{g}(x)=\varphi(x \rho(g))$. Since $\left(R_{\chi}^{\sim} \varphi\right)(x \rho(g))=\chi(g) \cdot R_{\chi}^{\sim}\left(\varphi^{g}\right)(x)\left(x \in V_{\mathbf{R}}, g \in G_{\mathbf{R}}\right)$, we have $R_{\chi}^{\sim} P^{s}(x \rho(g))=$ $\chi(g) \chi^{s}(g) \cdot P^{s}(g)$. Here $\chi^{s}$ stands for $\chi_{1}^{s_{1}} \cdots \chi_{n}^{s_{n}}$. The lemma easily follows from this formula. q.e.d.

Lemma 4.3. For $\chi, \chi^{\prime} \in X_{K}^{+}$, we have

$$
b_{\chi \chi^{\prime}}(s)=b_{\chi}(s) \cdot b_{\chi^{\prime}}(s+d(\chi)) .
$$

Proof. This is deduced from the formula $R_{x \chi^{\prime}}^{\sim}=R_{\chi^{\prime}}^{\sim} R_{\chi}^{\sim}$.

q.e.d.

We can define a rational function $b_{\chi}(s)$ of $s \in \mathbf{C}^{n}$ for any $\chi \in X_{K}$ preserving the relation (4.3). We call $b_{\chi}(s)$ the $b$-function of $\mathbf{D}$ corresponding to $\chi \in X_{K}$. The next results are proved in a similar manner as in $[5, \mathrm{Ch} . \mathrm{I}, \S 2]$.

Lemma 4.4. If $\chi \in X_{K}^{+}$, the highest homogeneous part of $b_{\chi}(s)$ is equal to $a_{\chi}(s)$ and $\operatorname{deg} b_{\chi}(s)=-\sum_{1 \leq i \leq n} \delta_{i} \cdot d(\chi)_{i}$.

Proposition 4.5. The notation and the assumption being the same as in Proposition 3.5, we have

$$
b_{\chi}(s)=c(\chi) \frac{\gamma(s)}{\gamma(s+d(\chi))}
$$


where $\gamma(s)=\Pi_{1 \leq j \leq m} \Pi_{k \in \mathbf{Z}} \Gamma\left(e_{j}(s)+k-C_{j}\right)^{n_{k}}$ with $C_{j} \in \mathbf{C}$ and $n_{k} \in \boldsymbol{Z}$. Here all but a finite number of $n_{k}$ are equal to zero.

\section{§5. Fourier transforms of modified complex powers of relative invariants}

In the rest of the paper, we let $\mathbf{D}=(G, V, \rho, \alpha)$ be a $\mathbf{Q}$-regular $\mathrm{PAD}$ and $\mathbf{D}^{*}=\left(G, V^{*}, \rho^{*}, \alpha^{*}\right)$ its dual. We further assume that the singular set $S$ of $\mathbf{D}$ is a hypersurface of $V$. Then the singular set $S^{*}$ of $\mathbf{D}^{*}$ is also a hypersurface of $V^{*}$. Thus $S$ (resp. $S^{*}$ ) is the disjoint union of the $\mathbf{Q}$-irreducible components $S_{1}, \ldots$, $S_{n}$ (resp. $\left.S_{1}^{*}, \ldots, S_{n}^{*}\right)$. Each $S_{i}$ (resp. $S_{i}^{*}$ ) is defined by a single equation $P_{i}(x)=0$ (resp. $P_{i}^{*}\left(x^{*}\right)=0$ ) with a $\mathbf{Q}$-irreducible polynomial $P_{i} \in \mathbf{Q}[V]$ (resp. $P_{i}^{*} \in$ $\left.\mathbf{Q}\left[V^{*}\right]\right)$. Denote by $\chi_{1}, \ldots, \chi_{n}\left(\right.$ resp. $\left.\chi_{1}^{*}, \ldots, \chi_{n}^{*}\right)$ be the corresponding characters to relative invariants $P_{1}, \ldots, P_{n}$ (resp. $\left.P_{1}^{*}, \ldots, P_{n}^{*}\right)$. For $s=\left(s_{1}, \ldots, s_{n}\right) \in \mathbf{C}^{n}$, we put

$$
\begin{gathered}
|P(x)|^{s}=\left|P_{1}(x)\right|^{s_{1}} \cdots\left|P_{n}(x)\right|^{s_{n}} \quad\left(x \in V_{\mathbf{R}}-S_{\mathbf{R}}\right) \\
\left|P^{*}\left(x^{*}\right)\right|^{s}=\left|P_{1}^{*}\left(x^{*}\right)\right|^{s_{1}} \cdots\left|P_{n}^{*}\left(x^{*}\right)\right|^{s_{n}} \quad\left(x^{*} \in V_{\mathbf{R}}^{*}-S_{\mathbf{R}}^{*}\right) .
\end{gathered}
$$

Let $V_{1}, \ldots, V_{\nu}$ (resp. $V_{1}^{*}, \ldots, V_{\nu}^{*}$ ) be the connected components of $V_{\mathbf{R}}-S_{\mathbf{R}}$ (resp. $V_{\mathbf{R}}^{*}-S_{\mathbf{R}}^{*}$ ). We here note that the the number of the connected components of $V_{\mathbf{R}}-S_{\mathbf{R}}$ coincides with that of $V_{\mathbf{R}}^{*}-S_{\mathbf{R}}^{*}$. Fix a normalized pair $\left(\beta, \beta^{*}\right)$ for $\left(\mathbf{D}, \mathbf{D}^{*}\right)$ defined over $\mathbf{Q}$. For $s \in \mathbf{C}^{n}$ and $i=1, \ldots, \nu$, we set

$$
\begin{gathered}
\Phi_{i}(f, s)=\int_{V_{i}}|P(x)|^{s} \mathbf{e}[\beta(x)] f(x) d x \quad\left(f \in \mathscr{S}\left(V_{\mathbf{R}}\right)\right) \\
\Phi_{i}^{*}\left(x^{*}\right)=\int_{V_{i}^{*}}\left|P^{*}\left(x^{*}\right)\right|^{s} \mathbf{e}\left[-\beta^{*}\left(x^{*}\right)\right] f^{*}\left(x^{*}\right) d x^{*} \quad\left(f^{*} \in \mathscr{\&}\left(V_{\mathbf{R}}^{*}\right)\right)
\end{gathered}
$$

Here $d x$ is a fixed Lebesgue measure on $V_{\mathbf{R}}$ and $d x^{*}$ denotes its dual measure on $V_{\mathbf{R}}^{*}$. The integrals $\Phi_{i}(f, s)$ and $\Phi_{i}^{*}\left(f^{*}, s\right)$ are absolutely convergent and define holomorphic functions in the region $\left\{s \in \mathbf{C}^{n} \mid \operatorname{Re} s_{1}>0, \ldots, \operatorname{Re} s_{n}>0\right\}$. Observe that, for a sufficiently large integer $r,\left(P_{1} \cdots P_{n}\right)^{r} \beta$ (resp. $\left.\left(P_{1}^{*} \cdots P_{n}^{*}\right)^{r} \beta^{*}\right)$ is a polynomial function on $V$ (resp. $V^{*}$ ). Then it is straightforward to show that $u(x)=\mathbf{e}[\beta(x)]$ (resp. $u^{*}\left(x^{*}\right)=\mathbf{e}\left[\beta^{*}\left(x^{*}\right)\right]$ ) satisfies the assumptions of Theorem A.3 in the appendix of [1]. Applying this theorem to our situation, we get the following result:

Proposition 5.1. Assume that the condition (3.6) is satisfied.

(i) The integrals $\Phi_{i}(f, s)$ and $\Phi_{i}^{*}(f, s)$ are continued to meromorphic functions of $s$ 
in $\mathbf{C}^{n}$.

(ii) There exist gamma factors $\Gamma_{\mathbf{D}}(s)$ and $\Gamma_{\mathbf{D}^{*}}(s)$ independent of $f$ and $f^{*}$ of the form

$$
\begin{aligned}
& \Gamma_{\mathbf{D}}(s)=\prod_{1 \leq i \leq m} \Gamma\left(a_{i 1} s_{1}+\cdots+a_{i n} s_{n}+b_{i}\right) \quad\left(a_{i j}, b_{i} \in \mathbf{C}\right), \\
& \Gamma_{\mathbf{D}^{*}}(s)=\prod_{1 \leq i \leq m} \Gamma\left(a_{i 1}^{*} s_{1}+\cdots+a_{i n}^{*} s_{n}+b_{i}^{*}\right) \quad\left(a_{i j}^{*}, b_{i}^{*} \in \mathbf{C}\right),
\end{aligned}
$$

such that $\Gamma_{\mathbf{D}}(s)^{-1} \Phi_{i}(f, s)$ and $\Gamma_{\mathbf{D}^{*}}(s)^{-1} \Phi_{i}^{*}\left(f^{*}, s\right)$ are entire functions.

(ii) The mappings $f \rightarrow \Phi_{i}(f, s)$ and $f^{*} \rightarrow \Phi_{i}^{*}\left(f^{*}, s\right)$ define tempered distributions depending meromorphically on $s \in \mathbf{C}^{n}$. If $Y_{0}$ is a bounded domain in $\mathbf{R}^{n}$ such that $\Phi_{i}(f, s)$ and $\Phi_{i}^{*}\left(f^{*}, s\right)$ are holomorphic in the tube domain $r=r_{0}+i \mathbf{R}^{n}$, then the orders of these tempered distributions are bounded for $s \in Y$.

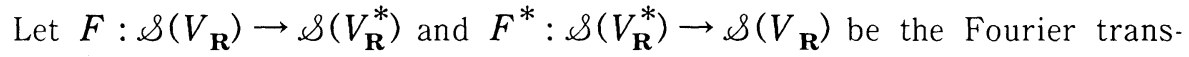
forms given by

$$
\begin{array}{cc}
F f\left(x^{*}\right)=\int_{V_{\mathbf{R}}} f(x) \mathbf{e}\left[-\left\langle x, x^{*}\right\rangle\right] d x & \left(f \in \mathscr{S}\left(V_{\mathbf{R}}\right), x^{*} \in V_{\mathbf{R}}^{*}\right) \\
F^{*} f^{*}(x)=\int_{V_{\mathbf{R}}^{*}} f^{*}\left(x^{*}\right) \mathbf{e}\left[\left\langle x, x^{*}\right\rangle\right] d x^{*} & \left(f^{*} \in \mathscr{S}\left(V_{\mathbf{R}}^{*}\right), x \in V_{\mathbf{R}}\right) .
\end{array}
$$

Recall that $\chi_{0}(g)=(\operatorname{det} a(g))^{2} \in X_{\rho}(G)_{\mathbf{Q}}$ (see Lemma 2.7). Put

$$
\lambda=\frac{1}{2} d\left(\chi_{0}\right) \in\left(\frac{1}{2} \mathbf{Z}\right)^{n}, \lambda^{*}=\frac{1}{2} d^{*}\left(\chi_{0}^{-1}\right) \in\left(\frac{1}{2} \mathbf{Z}\right)^{n},
$$

Then we have $\lambda^{*}=-\lambda U$ (cf. (3.1)). Let $\gamma(s)$ and $c(\chi)$ be as in Proposition 4.5. We set

$$
\begin{gathered}
c(s)=c\left(\chi_{1}\right)^{s_{1}} \cdots c\left(\chi_{n}\right)^{s_{n}} \\
\Phi(f, s)={ }^{t}\left(\Phi_{1}(f, s), \ldots, \Phi_{\nu}(f, s)\right) \\
\Phi^{*}\left(f^{*}, s\right)={ }^{t}\left(\Phi_{1}^{*}\left(f^{*}, s\right), \ldots, \Phi_{\nu}^{*}\left(f^{*}, s\right)\right)
\end{gathered}
$$

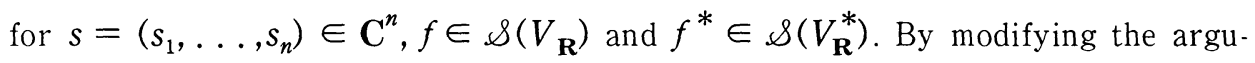
ment in $[3, \S 5]$ in a suitable manner, we can prove

THEOREM 5.2. The following functional equation holds:

$$
\Phi\left(F^{*} f^{*}, s\right)=c(-s) \gamma(s) A(s) \Phi^{*}\left(f^{*},(s+\lambda) U\right),
$$


where $A(s)=\left(A_{i j}(s)\right)$ is a $\nu \times \nu$ matrix whose entries are polynomials in $\exp ( \pm$ $\left.\pi i s_{1}\right), \ldots, \exp \left( \pm \pi i s_{n}\right)$.

\section{\$6. Zeta functions attached to a Q-regular PAD}

We keep the notation and the assumptions in the previous section. In this section, we further assume that

$$
x \in V_{\mathbf{Q}}-S_{\mathbf{Q}} \Rightarrow X\left(G(x)^{0}\right)_{\mathbf{Q}}=\{1\},
$$

where $G(x)^{0}$ is the identity component of $G(x)$. This assumption implies $X\left(G\left(x^{*}\right)^{0}\right)=\{1\}$ for $x^{*} \in V_{\mathbf{Q}}^{*}-S_{\mathbf{Q}}^{*}$.

We take a basis of $V$ and a matrix expression of $G$ so that they are compatible with the $\mathbf{Q}$-structure of $\mathbf{D}$. Let $\Gamma=G_{\mathbf{Z}} \cap G_{\mathbf{R}}^{+}$be a discrete subgroup of $G_{\mathbf{R}}^{+}$. A lattice $L$ in $V_{\mathbf{Q}}$ is said to be $\Gamma$-admissible with respect to $(\rho, \alpha)$ if $L \cdot \rho(\Gamma) \subset L$ and if $\alpha(L, \Gamma) \subset \mathbf{Z}$. The following lemma is easily verified.

Lemma 6.1. Let $L$ be a lattice of $V_{\mathbf{Q}}$ and $L^{*}=\left\{x^{*} \in V_{\mathbf{Q}}^{*} \mid\left\langle x, x^{*}\right\rangle \in \mathbf{Z}\right.$ for any $x \in L\}$ be its dual. Then $L$ is $\Gamma$-admissible with respect to $(\rho, \alpha)$ if and only if $L^{*}$ is $\Gamma$-admissible with respect to $\left(\rho^{*}, \alpha^{*}\right)$.

Define $G_{\mathbf{R}}^{+}$-invariant measures $\omega$ and $\omega^{*}$ on $V_{\mathbf{R}}-S_{\mathbf{R}}$ and $V_{\mathbf{R}}^{*}-S_{\mathbf{R}}^{*}$ by $\omega(x)=|P(x)|^{-\lambda} d x, \omega^{*}\left(x^{*}\right)=\left|P^{*}\left(x^{*}\right)\right|^{-\lambda^{*}} d x^{*}$ (for the definition of $\lambda$ and $\lambda^{*}$, see (5.7)). We put

$$
\begin{gathered}
G(x)^{+}=G(x) \cap G_{\mathbf{R}}^{+}, \Gamma(x)=G(x)^{+} \cap \Gamma \quad\left(x \in V_{\mathbf{Q}}-S_{\mathbf{Q}}\right) \\
G^{*}\left(x^{*}\right)^{+}=G^{*}\left(x^{*}\right) \cap G_{\mathbf{R}}^{+}, \Gamma^{*}\left(x^{*}\right)=G^{*}\left(x^{*}\right)^{+} \cap \Gamma \quad\left(x^{*} \in V_{\mathbf{Q}}^{*}-S_{\mathbf{Q}}^{*}\right) .
\end{gathered}
$$

Under the assumption (6.1), $\operatorname{vol}\left(\Gamma(x) \backslash G(x)^{+}\right)$and $\operatorname{vol}\left(\Gamma^{*}\left(x^{*}\right) \backslash G^{*}\left(x^{*}\right)^{+}\right)$are finite. From now on we fix a right invariant Haar measure $d g$ on $G_{\mathbf{R}}^{+}$. For $x \in V_{\mathbf{Q}}$ $-S_{\mathbf{Q}}$ (resp. $x^{*} \in V_{\mathbf{Q}}^{*}-S_{\mathbf{Q}}^{*}$ ), we normalize the Haar measure $d m_{x}$ (resp. $d m_{x^{*}}$ ) on $G(x)^{+}$(resp. $\mathrm{G}^{*}\left(x^{*}\right)^{+}$) by

$$
\begin{aligned}
& \int_{G_{\mathbf{R}}^{+}} \varphi(g) d g=\int_{G(x)^{+} \backslash G_{\mathbf{R}}^{+}} \omega(x \rho(g)) \int_{G(x)^{+}} \varphi(h g) d m_{x}(h) \\
& =\int_{G^{*}\left(x^{*}\right)^{+} \backslash G_{\mathbf{R}}^{+}} \omega^{*}\left(x^{*} \rho^{*}(g)\right) \int_{G^{*}\left(x^{*}\right)^{+}} \varphi(h g) d m_{x^{*}}(h) \quad\left(\varphi \in C_{c}^{\infty}\left(G_{\mathbf{R}}^{+}\right)\right) .
\end{aligned}
$$

We define the density $\mu(x)$ (resp. $\mu^{*}\left(x^{*}\right)$ ) at $x \in V_{\mathbf{Q}}-S_{\mathbf{Q}}$ (resp. $x^{*} \in V_{\mathbf{Q}}^{*}-$ $\left.S_{\mathbf{Q}}^{*}\right)$ to be 


$$
\mu(x)=\int_{\Gamma(x) \backslash G(x)^{+}} d m_{x}(h), \mu^{*}\left(x^{*}\right)=\int_{\Gamma^{*}\left(x^{*}\right) \backslash G^{*}\left(x^{*}\right)^{+}} d m_{x^{*}}(h) .
$$

Let $L$ be a $\Gamma$-admissible lattice of $V_{\mathbf{R}}$ with respect to $(\rho, \alpha)$ and $L^{*}$ its dual. Put $L^{\prime}=L \cap\left(V_{\boldsymbol{R}}-S_{\mathbf{R}}\right), L^{* \prime}=L^{*} \cap\left(V_{\mathbf{R}}^{*}-S_{\mathbf{R}}^{*}\right), L_{i}=L \cap V_{i}$ and $L_{i}^{*}=L^{*} \cap$ $V_{i}^{*}(1 \leq i \leq \nu)$. Then $L^{\prime}=\cup_{1 \leq i \leq \nu} L_{i}$ and $L^{* \prime}=U_{1 \leq i \leq \nu} L_{i}^{*}$ (disjoint union). We now define the zeta functions $\xi_{i}(s, L)$ and $\xi_{i}^{*}\left(s, L^{*}\right)$ by the following Dirichlet series:

$$
\begin{gathered}
\xi_{i}(s, L)=\sum_{x \in L_{i} / \rho(\Gamma)} \mu(x) \mathbf{e}[-\beta(x)]|P(x)|^{-s} \\
\xi_{i}^{*}\left(s, L^{*}\right)=\sum_{x \in L_{i}^{*} / \rho^{*}(\Gamma)} \mu^{*}\left(x^{*}\right) \mathbf{e}\left[\beta^{*}\left(x^{*}\right)\right]\left|P^{*}\left(x^{*}\right)\right|^{-s} .
\end{gathered}
$$

Henceforth we always assume

(6.5) The Dirichlet series $\xi_{1}(s, L), \ldots, \xi_{\nu}(s, L)\left(\right.$ resp. $\left.\xi_{1}^{*}\left(s, L^{*}\right), \ldots, \xi_{\nu}^{*}\left(s, L^{*}\right)\right)$ are absolutely convergent for $\operatorname{Re}\left(s_{1}\right)>a_{1}, \ldots, \operatorname{Re}\left(s_{n}\right)>a_{n}\left(\right.$ resp. $\operatorname{Re}\left(s_{1}\right)>a_{1}^{*}, \ldots$, $\left.\operatorname{Re}\left(s_{n}\right)>a_{n}^{*}\right)$, where $a_{i}, a_{i}^{*}(1 \leq i \leq n)$ are certain positive real numbers.

Set $B=\left\{s \in \mathbf{C}^{n} \mid \operatorname{Re} s_{i}>\operatorname{Max}\left(a_{i}, \lambda_{i}\right)(1 \leq i \leq n)\right\}$ and $B^{*}=\left\{s \in \mathbf{C}^{n} \mid\right.$

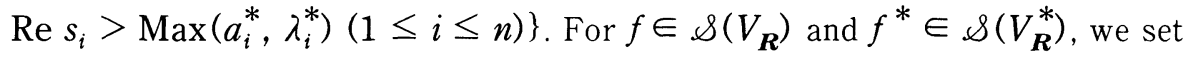

$$
\begin{gathered}
Z(s, f, L)=\int_{\Gamma \backslash G_{\mathbf{R}}^{+}}|\chi(g)|^{s-\lambda / 2} \sum_{x \in L^{\prime}} r(g) f(x) d g \\
Z^{*}\left(s, f^{*}, L^{*}\right)=\int_{\Gamma \backslash G_{\mathbf{R}}^{+}}\left|\chi^{*}(g)\right|^{s-\lambda^{*} / 2} \sum_{x^{*} \in L^{*^{\prime}}} r^{*}(g) f^{*}\left(x^{*}\right) d g,
\end{gathered}
$$

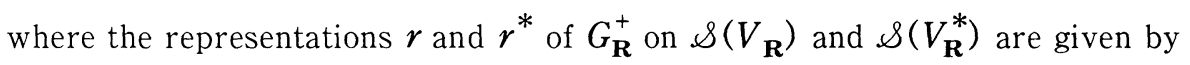

$$
\begin{gathered}
r(g) f(x)=f(x \rho(g)) \mathbf{e}[\alpha(x, g)]\left(\frac{d(x \rho(g))}{d x}\right)^{1 / 2} \\
r^{*}(g) f^{*}\left(x^{*}\right)=f^{*}\left(x^{*} \rho^{*}(g)\right) \mathbf{e}\left[-\alpha^{*}\left(x^{*}, g\right)\right]\left(\frac{d\left(x^{*} \rho^{*}(g)\right)}{d x^{*}}\right)^{1 / 2}
\end{gathered}
$$

$\left(x \in V_{\mathbf{R}}, x^{*} \in V_{\mathbf{R}}^{*}, f \in \mathscr{S}\left(V_{\mathbf{R}}\right), f^{*} \in \mathscr{S}\left(V_{\mathbf{R}}^{*}\right)\right)$. It is easy to see that

$$
F \cdot r(g)=r^{*}(g) \cdot F, r(g) \cdot F^{*}=F^{*} \cdot r^{*}(g) \quad\left(g \in G_{\mathbf{R}}^{+}\right) .
$$

The following lemma is easily verified.

Lemma 6.2. The integrals $Z(s, f, L)$ and $Z^{*}\left(s, f^{*}, L^{*}\right)$ are absolutely convergent for $s \in B$ and $s \in B^{*}$, respectively. Furthermore we have 


$$
\begin{gathered}
Z(s, f, L)=\sum_{1 \leq i \leq \nu} \xi_{i}(s, L) \Phi_{i}(f, s-\lambda) \\
Z^{*}\left(s, f^{*}, L^{*}\right)=\sum_{1 \leq i \leq \nu} \xi_{i}^{*}\left(s, L^{*}\right) \Phi_{i}^{*}\left(f^{*}, s-\lambda^{*}\right) .
\end{gathered}
$$

Let $\Omega$ (resp. $\Omega^{*}$ ) be the convex hull of $\left(B^{*} U^{-1}+\lambda\right) \cup B$ (resp. $(B-\lambda) U$ $\left.\cup B^{*}\right)$ in $\mathbf{C}^{n}$. Then $(\Omega-\lambda) U=\Omega^{*}$. Applying the Poisson summation formula and using (6.10), we obtain the following:

THEOREM 6.3. Assume that $f^{*} \in \mathscr{S}\left(V_{\mathbf{R}}^{*}\right)$ and its Fourier transform $F^{*} f^{*}$ vanish on $S_{\mathbf{R}}^{*}$ and $S_{\mathbf{R}}$, respectively. Then $Z\left(s, F^{*} f^{*}, L\right)$ and $Z^{*}\left(s, f^{*}, L^{*}\right)$ are continued to $\Omega$ and $\Omega^{*}$ respectively as meromorphic functions of $s$. Furthermore the following functional equation holds:

$$
Z^{*}\left((s-\lambda) U, f^{*}, L^{*}\right)=\operatorname{vol}\left(V_{\mathbf{R}}^{*} / L^{*}\right)^{-1} Z\left(s, F^{*} f^{*}, L\right) \quad(s \in \Omega) .
$$

For simplicity, we write $b_{0}(s)$ and $b_{0}^{*}(s)$ for $b_{x_{1}^{*} \cdots x_{n}^{*}}(s)$ and $b_{x_{1} \cdots x_{n}}^{*}(s)$, where $b_{x^{*}}(s)$ (resp. $b_{\chi}^{*}(s)$ ) is the $b$-function of $\mathbf{D}$ (resp. $\mathbf{D}^{*}$ ). We can now state our main result, which follows from Theorem 5.2 and Theorem 6.3 in use of the standard argument in the theory of zeta functions of PV's (for example, see [8] and [3]).

THEOREM 6.4. Assume that the conditions (3.6), (6.1) and (6.5) are satisfied. (i) The Dirichlet series $\xi_{1}(s, L), \ldots, \xi_{\nu}(s, L)$ (resp. $\left.\xi_{1}^{*}\left(s, L^{*}\right), \ldots, \xi_{\nu}^{*}\left(s, L^{*}\right)\right)$ are continued to meromorphic functions of $s$ on $\Omega$ (resp. $\left.\Omega^{*}\right)$. Furthermore $b_{0}(s-\lambda)$. $\xi_{i}(s, L)\left(\right.$ resp. $\left.b_{0}^{*}\left(s-\lambda^{*}\right) \cdot \xi_{i}^{*}\left(s, L^{*}\right)\right)$ is holomorphic on $\Omega\left(\right.$ resp. $\left.\Omega^{*}\right)$ for $i=1, \ldots, \nu$. (ii) The following functional equations hold for $i=1, \ldots, \nu$ :

$$
\operatorname{vol}\left(V_{\mathbf{R}}^{*} / L^{*}\right) \cdot \xi_{i}^{*}\left((s-\lambda) U, L^{*}\right)=c(\lambda-s) \gamma(s-\lambda) \sum_{1 \leq j \leq \nu} A_{j i}(s-\lambda) \cdot \xi_{j}(s, L),
$$

where $s \in \Omega$ and $A_{j i}(s)$ is a polynomial in $\exp \left( \pm \pi i s_{1}\right), \ldots, \exp \left( \pm \pi i s_{n}\right)$ given in Theorem 5.2 .

\section{\$7. Examples}

1. Let $\mathbf{D}=(G, V, \rho, \alpha)$ be an affine datum over $\mathbf{Q}$. For $a \in V_{\mathbf{Q}}$ and $b^{*} \in$ $V_{\mathbf{Q}^{\prime}}^{*}$ define an algebraic homomorphism $\rho_{a, b^{*}}: G \rightarrow \operatorname{Aff}(V)$ and an affine 1-cocycle $\alpha_{a, b^{*}}: V \times G \rightarrow \mathbf{G}_{a}$ by

$$
\begin{aligned}
& x \rho_{a, b^{*}}(g)=(x+a) \rho(g)-a, \\
& \alpha_{a, b^{*}}(x, g)=\alpha(x+a, g)+\left\langle(x+a)\left(\rho(g)-1_{V}\right), b^{*}\right\rangle .
\end{aligned}
$$


Then $\mathbf{D}_{a, b^{*}}=\left(G, V, \rho_{a, b^{*}}, \alpha_{a, b^{*}}\right)$ is also an affine datum, which we call the shift of $\mathbf{D}$ by $\left(a, b^{*}\right)$. If $\mathbf{D}$ is a PAD with singular set $S$, then $\mathbf{D}_{a, b^{*}}$ is also prehomogeneous and its singular set is $S_{a}=\{x \in V \mid x+a \in S\}$. The dual of $\mathbf{D}_{a, b^{*}}$ is the shift $\mathbf{D}_{b^{*}, a}^{*}$ of the dual $\mathbf{D}^{*}$ of $\mathbf{D}$ by $\left(b^{*}, a\right)$. It is easily verified that $\mathbf{D}_{a, b^{*}}$ is regular if and only if so is $\mathbf{D}$.

Let $\mathbf{D}$ be a $\mathbf{Q}$-regular PAD satisfying the conditions (3.6) and (6.1). Then its shift $\mathbf{D}_{a, b^{*}}$ is also $\mathbf{Q}$-regular and satisfies (3.6) and (6.1). Let $\left(\beta, \beta^{*}\right)$ be a normalized pair for $\left(\mathbf{D}, \mathbf{D}^{*}\right.$ ) defined over $\mathbf{Q}$ (for definition, see $\S 3$ ). Put

$$
\begin{gathered}
\beta_{a, b^{*}}(x)=\beta(x+a)+\left\langle x+\frac{1}{2} a, b^{*}\right\rangle \quad(x \in V), \\
\beta_{b^{*}, a}^{*}\left(x^{*}\right)=\beta^{*}\left(x^{*}+b^{*}\right)+\left\langle a, x^{*}+\frac{1}{2} b^{*}\right\rangle \quad\left(x^{*} \in V^{*}\right) .
\end{gathered}
$$

Then $\left(\beta_{a, b^{*}}, \beta_{b^{*}, a}^{*}\right)$ is a normalized pair for $\left(\mathbf{D}_{a, b^{*}}, \mathbf{D}_{b^{*}, a}^{*}\right)$ defined over $\mathbf{Q}$. We can easily verify that the $a$-function, the $b$-function and the data appearing in Theorem $5.2(\lambda, U, c(s), \gamma(s)$ and $A(s))$ do not change for the shifting of PAD. Thus zeta functions attached to $\mathbf{D}_{a, b^{*}}$ have the same functional equations as those attached to $\mathbf{D}$.

2. We now consider the simplest $\operatorname{PAD} \mathbf{D}=(G, V, \rho, \alpha)$ with $G_{\mathbf{Q}}=\mathbf{Q}^{\times}$, $V_{\mathbf{Q}}=\mathbf{Q}, x \rho(t)=x t$ and $\alpha(x, t)=0\left(x \in \mathbf{Q}, t \in \mathbf{Q}^{\times}\right)$. The singular set is $S=$ $\{0\}$ and $V_{\mathbf{R}}-S_{\mathbf{R}}=V_{1} \cup V_{2}$, where $V_{1}=\{x \in \mathbf{R} \mid x>0\}$ and $V_{2}=\{x \in \mathbf{R} \mid$ $x<0\}$. We identify $V$ and $V^{*}$ via the inner product $\langle x, y\rangle=x y$. In this case, the data appearing in Theorem 5.2 are given by

$$
\begin{aligned}
& \lambda=1, U=(-1), c(s)=(2 \pi)^{s} \mathbf{e}\left[-\frac{s}{4}\right], \gamma(s)=\Gamma(s+1), \\
& A(s)=\frac{-1}{2 \pi i}\left(\begin{array}{cc}
1 & -\mathbf{e}[-s / 2] \\
-\mathbf{e}[-s / 2] & 1
\end{array}\right) .
\end{aligned}
$$

Let $\mathbf{D}_{a, b}$ be the shift of $\mathbf{D}$ by $a$ and $b(a, b \in \mathbf{Q})$. Since $\Gamma=G_{\mathbf{Z}}^{+}=\{1\}, L=\mathbf{Z}$ is a $\Gamma$-admissible lattice of $\mathbf{D}_{a, b}$ and associated zeta functions are given as follows:

$$
\begin{aligned}
& \xi_{1}(s, L)=\mathbf{e}\left[-\frac{a b}{2}\right] \zeta_{+}(s, a,-b), \xi_{2}(s, L)=\mathbf{e}\left[-\frac{a b}{2}\right] \zeta_{-}(s, a,-b), \\
& \xi_{1}^{*}\left(s, L^{*}\right)=\mathbf{e}\left[\frac{a b}{2}\right] \zeta_{+}(s, b, a), \xi_{2}^{*}\left(s, L^{*}\right)=\mathbf{e}\left[\frac{a b}{2}\right] \zeta_{-}(s, b, a),
\end{aligned}
$$

Here $\zeta_{ \pm}(s, a, b)$ are the usual Hurwitz-Lerch zeta functions given by 


$$
\zeta_{ \pm}(s, a, b)=\sum_{n \in \mathbf{Z}, \pm(n+a)>0} \mathbf{e}[b n]|n+a|^{-s} .
$$

Then Theorem 6.4 implies the well-known functional equations of Hurwitz-Lerch zeta functions:

$$
\begin{aligned}
& \left(\zeta_{+}(1-s, b, a), \zeta_{-}(1-s, b, a)\right) \\
& =\mathbf{e}[-a b](2 \pi)^{-s} \Gamma(s)\left(\zeta_{+}(s, a,-b), \zeta_{-}(s, a,-b)\right) \times\left(\begin{array}{cc}
\mathbf{e}[s / 4] & \mathbf{e}[-s / 4] \\
\mathbf{e}[-s / 4] & \mathbf{e}[s / 4]
\end{array}\right) .
\end{aligned}
$$

Remark. Zeta functions associated with a shift of a regular PV are considered as a generalization of the Hurwitz-Lerch zeta functions. Such zeta functions have been studied by F. Sato from the point of view different from ours (see [4, $\S 4])$. In fact, he has shown their functional equations by using his theory of zeta distributions.

3. Let $n$ and $m$ be positive integers and put $V=\operatorname{Sym}_{n}(=$ the space of symmetric matrices of degree $n)$ and $W=\mathrm{M}_{m, n}$. Let $\mathbf{G}=\{\xi, g)=\left(\begin{array}{cc}1_{m} & \xi \\ 0 & 1_{n}\end{array}\right)$. $\left.\left(\begin{array}{cc}1_{m} & 0 \\ 0 & g\end{array}\right) \mid \xi \in W, g \in \mathrm{GL}_{n}\right\}$ and $\mathbf{V}=V \times W$. We define an algebraic homomorphism $\rho$ of $\mathbf{G}$ into $\mathrm{GL}(\mathbf{V})$ by

$$
[x, u] \rho((\xi, g))=\left[g^{-1} x^{t} g^{-1},(u-\xi x)^{t} g^{-1}\right]
$$

for $[x, u] \in \boldsymbol{V}$ and $(\xi, g) \in \mathbf{G}$. The triplet $(\mathbf{G}, \mathbf{V}, \rho)$ is a non-regular PV with singular set $\mathbf{S}=\{[x, u] \in \mathbf{V} \mid P(x, u):=\operatorname{det}(x)=0\}$. Fix a positive definite semiintegral symmetric matrix $S$ of degree $m$ and define an affine 1-cocycle $\alpha$ by $\alpha([x, u],(\xi, g))=\operatorname{tr}\left(x^{t} \xi S \xi-2^{t} \xi S u\right)$. Then it is easily verified that $\mathbf{D}=$ $(\mathbf{G}, \mathbf{V}, \rho, \alpha)$ is a regular PAD. In this case, a function $\beta$ on $\mathbf{V}-\mathbf{S}$ satisfying (2.1) is given by $\beta([x, u])=\operatorname{tr}\left(x^{-1} \cdot{ }^{t} u S u\right)$. Note that $\mathbf{D}$ is not a shift of any PV. Identifying $\mathbf{V}^{*}$ with $\mathbf{V}$ via the inner product $\langle[x, u],[y, v]\rangle=\operatorname{tr}\left(x y+2^{t} u S v\right)([x, u]$, $[y, v] \in \mathbf{V})$, we see that the dual of $\mathbf{D}$ is given by $\mathbf{D}^{*}=\left(\mathbf{G}, \mathbf{V}, \rho^{*}, \alpha^{*}\right)$ where

$$
[x, u] \rho^{*}((\xi, g))=\left[{ }^{t} g\left(x+{ }^{t} \xi S \xi+{ }^{t} u S \xi+{ }^{t} \xi S u\right) g,(u+\xi) g\right], \alpha^{*} \equiv 0 .
$$

The singular set $\mathbf{S}^{*}$ of $\mathbf{D}^{*}$ is $\left\{[x, u] \in \mathbf{V} \mid P^{*}(x, u):=\operatorname{det}\left(x-{ }^{t} u S u\right)=0\right\}$. Note that the relative invariant $P^{*}(x, u)$ of $\mathbf{D}^{*}$ is not homogeneous. The associated zeta functions with $\mathbf{D}$ and $\mathbf{D}^{*}$ are the same ones as studied in [2] (for the precise form of their functional equations, see [2 Theorem 2.4]). Note that certain special values of the above zeta functions appear in the dimension formula for the space of Jacobi 
forms of degree $n$ (see [2, Theorem 4.3]).

4. Let $K$ be an imaginary quadratic field $o_{K}$ be the integer ring of $K$. We denote by $\bar{a}$ the conjugate of $a \in K$ and put $x^{*}={ }^{t} \bar{x}$ for $x \in \mathrm{M}_{r}(K)$. Let $\tau$ be the trace of $K$ to $\mathbf{Q}: \tau(a)=a+\bar{a}(a \in K)$. For positive integers $n$ and $m$, let $V_{\mathbf{Q}}=$ $\left\{x \in \mathbf{M}_{n}(K) \mid x^{*}=x\right\}, W_{\mathbf{Q}}=\mathbf{M}_{m, n}(K)$ and $\mathbf{V}_{\mathbf{Q}}=V_{\mathbf{Q}} \times W_{\mathbf{Q}}$. The group $\mathbf{G}_{\mathbf{Q}}=$ $\left\{(\xi, g)=\left(\begin{array}{cc}1_{m} & \xi \\ 0 & 1_{n}\end{array}\right)\left(\begin{array}{cc}1_{m} & 0 \\ 0 & g\end{array}\right) \mid \xi \in W_{\mathbf{Q}}, g \in \mathrm{GL}_{n}(K)\right\}$ acts on $\mathbf{V}_{\mathbf{Q}}$ by

$$
[x, u] \rho((\xi, g))=\left[g^{-1} x g^{*-1},(u-\xi x) g^{*-1}\right) \quad\left([x, u] \in \mathbf{V}_{\mathbf{Q}},(\xi, g) \in \mathbf{G}_{\mathbf{Q}}\right) .
$$

We fix a positive definite Hermitian matrix $H=\left(h_{i j}\right)$ of degree $m$ with $h_{i i}=\mathbf{Z}$ and $h_{i j} \in \delta_{K}^{-1}$, where $\delta_{K}$ is the different of $K$ over $\mathbf{Q}: \delta_{K}^{-1}=\left\{a \in K \mid \tau\left(a o_{K}\right) \subset \mathbf{Z}\right\}$. For $u, v \in W_{\mathbf{Q}}, H[u]$ and $H(u, v)$ stand for $u^{*} H u$ and $u^{*} H v$, respectively. For $[x, u] \in \mathbf{V}_{\mathbf{Q}}$ and $(\xi, g) \in \mathbf{G}_{\mathbf{Q}}$, put $\alpha([x, u],(\xi, g))=\operatorname{tr}(x \cdot H[\xi])-\tau(\operatorname{tr}(H(\xi, u)))$. Then $\alpha$ is an affine 1 -cocycle with respect to $\rho$. It is easily verified that $\mathbf{D}=(\mathbf{G}, \mathbf{V}, \rho$, $\alpha)$ is a PAD defined over $\mathbf{Q}$ with singular set $\mathbf{S}=\{[x, u] \in \mathbf{V} \mid P([x, u]):=$ $\operatorname{det} x=0$. Henceforth we identify the $\mathbf{Q}$-dual $\mathbf{V}_{\mathbf{Q}}^{*}$ of $\mathbf{V}_{\mathbf{Q}}$ with $\mathbf{V}_{\mathbf{Q}}$ itself via the inner product $\langle$,$\rangle of \mathbf{V}_{\mathbf{Q}}$ defined by $\langle[x, u],[y, v]\rangle=\operatorname{tr}(x y)+\tau(\operatorname{tr}(H(u, v)))$. Then the dual $\mathbf{D}^{*}=\left(\mathbf{G}, \mathbf{V}, \rho^{*}, \alpha^{*}\right)$ is given by $[x, u] \rho^{*}((\xi, g))=\left[g^{*}(x+H[u+\xi]-\right.$ $H[u]) g,(u+\xi) g], \alpha^{*} \equiv 0$. We easily see that $\mathbf{D}^{*}$ is also PAD and its singular set is $\mathbf{S}^{*}=\left\{[x, u] \in \mathbf{V} \mid P^{*}([x, u]):=\operatorname{det}(x-H[u])=0\right\}$. The corresponding character $\chi_{1}\left(\right.$ resp. $\left.\chi_{1}^{*}\right)$ to $P\left(\right.$ resp. $\left.P^{*}\right)$ is given by $\chi_{1}((\xi, g))=\mathrm{N}_{K / \mathbf{Q}}(\operatorname{det} g)^{-1}\left(\right.$ resp. $\chi_{1}^{*}((\xi, g))$ $\left.=\mathrm{N}_{K / \mathbf{Q}}(\operatorname{det} g)\right)$. Thus $\mathbf{G}_{1, \mathbf{Q}}=\left\{(\xi, g) \in \mathbf{G}_{\mathbf{Q}} \mid \mathbf{N}_{K / \mathbf{Q}}(\operatorname{det} g)=1\right\}$. For simplicity we write $(\xi, A)$ for each element $\left(\begin{array}{ll}0 & \xi \\ 0 & A\end{array}\right)$ of the Lie algebra $g_{\mathbf{Q}}$ of $\mathbf{G}_{\mathbf{Q}}\left(\xi \in \mathrm{M}_{m, n}(K), A \in\right.$ $\left.\mathrm{M}_{n}(K)\right)$. Then the differentials of $\rho, \alpha$ and $\chi_{1}$ are given as follows:

$$
\begin{aligned}
& {[x, u] d \rho((\xi, A))=\left[-A x-x A^{*},-\xi x-u A^{*}\right],} \\
& d \alpha([x, u](\xi, A))=-\tau(\operatorname{tr} H(\xi, u)), d \chi_{1}((\xi, A))=-\tau(\operatorname{tr} A) .
\end{aligned}
$$

Since $d \chi_{1}$ vanishes on the Lie algebra $\mathfrak{g}_{1}$ of $\mathbf{G}_{1}$, we can define a $\mathbf{G}$-equivariant mapping $\phi_{s}: \mathbf{V}-\mathbf{S} \rightarrow \mathbf{V}-\mathbf{S}^{*}$ for $s \in \mathbf{C}$ so that

$$
\left\langle[x, u] d \rho((\xi, A)), \phi_{s}([x, u])\right\rangle+d \alpha([x, u],(\xi, A))=-\frac{1}{2 \pi i} s d \chi_{1}((\xi, A))
$$

for every $[x, u] \in \mathbf{V}$ and $(\xi, A) \in \mathrm{g}_{\mathbf{Q}}$ (see Proposition 2.3). In fact, we have $\phi_{s}([x, u])=\left[-\frac{s}{2 \pi i} x^{-1}+H\left[u x^{-1}\right],-u x^{-1}\right]$ and hence $\phi_{s}$ is dominant if $s \neq 0$. Thus $\mathbf{D}$ and $\mathbf{D}^{*}$ are $\mathbf{Q}$-regular. We normalize $\beta$ and $\beta^{*}$ satisfying (2.1) by 
$\beta([x, u])=\operatorname{tr}\left(x^{-1} H[u]\right), \beta^{*} \equiv 0$. Then $F(s)$ defined in $\S 3$ is equal to $-\frac{n}{2 \pi i} s$ and hence the condition (3.6) is satisfied.

Let $V_{i}$ be the set of $n \times n$ Hermitian matrices with $i$ positive and $n-i$ negative eigenvalues $(0 \leq i \leq n)$. Put $\mathbf{V}_{i}=V_{i} \times W_{\mathbf{R}}$ and $\mathbf{V}_{i}^{*}=\left\{[x, u] \in \mathbf{V}_{\mathbf{R}} \mid x-H[u] \in\right.$ $V_{i}$ ). Then we have $\mathbf{V}_{\mathbf{R}}-\mathbf{S}_{\mathbf{R}}=\cup_{i=0}^{n} \mathbf{V}_{i}$ and $\mathbf{V}_{\mathbf{R}}-\mathbf{S}_{\mathbf{R}}^{*}=\cup_{i=0}^{n} \mathbf{V}_{i}^{*}$ (disjoint union). Let $d x=\Pi_{i=1}^{n} d x_{i i} \Pi_{i<j} d \operatorname{Re}\left(x_{i j}\right) d \operatorname{Im}\left(x_{i j}\right)\left(x=\left(x_{i j}\right) \in V_{\mathbf{R}}\right)$ and $\quad d u=\Pi_{i=1}^{m} \Pi_{j=1}^{n} d \operatorname{Re}\left(u_{i j}\right)$ $d \operatorname{Im}\left(u_{i j}\right)$ be the usual Lebesgue measures on $V_{\mathbf{R}}$ and $W_{\mathbf{R}}$, respectively. For $f \in \mathscr{S}\left(\mathbf{V}_{\mathbf{R}}\right)$, define

$$
\begin{aligned}
& \Phi_{i}(f, s)=\int_{\mathbf{V}_{i}} f([x, u])|\operatorname{det} x|^{s} \mathbf{e}\left[\operatorname{tr}\left(x^{-1} H[u]\right)\right] d x d u, \\
& \Phi_{i}^{*}(f, s)=\int_{\mathbf{V}_{i}^{*}} f([x, u])|\operatorname{det}(x-H[u])|^{s} d x d u .
\end{aligned}
$$

Both functions $\Phi_{i}(f, s)$ and $\Phi_{i}^{*}(f, s)$ are continued to meromorphic functions on $\mathbf{C}$ (Proposition 5.1). Let $F^{*}$ be the Fourier transform of $f \in \Theta\left(\mathbf{V}_{\mathbf{R}}\right)$ :

$$
F^{*} f([x, u])=\int_{\mathbf{V}_{\mathbf{R}}} f([y, v]) \mathbf{e}[\langle[x, u],[y, v]\rangle] d y d v
$$

The following functional equation is proved by a similar argument to that of [2, Proposition 1.4$]$ and by using the results of $[8, \S 4]$.

THEOREM 7.1. For $f \in \mathscr{S}\left(\mathbf{V}_{\mathbf{R}}\right)$, we have

$$
\Phi_{i}\left(F^{*} f, s\right)=c(-s) \gamma(s) \sum_{j=0}^{n} A_{i j}(s) \Phi_{j}^{*}(f,-s-m-n),
$$

where

$$
\begin{gathered}
c(s)=\mathbf{e}\left[-\frac{n s}{4}\right](2 \pi)^{n s}, \gamma(s)=\prod_{1 \leq i \leq n} \Gamma(s+m+i), \\
A_{i j}(s)=(\operatorname{det} 2 H)^{-n} \mathbf{e}\left[\frac{n^{2}-2 m i}{4}\right](2 \pi)^{-n(m+n)} u_{i j}(s+m+n),
\end{gathered}
$$

with $u_{i j}(s)=(-1)^{(n-i)(n-1)} \pi^{n(n-1) / 2} \times \sum_{k=\operatorname{Max}(0, i+j-n)}^{\operatorname{Min}(i, j)}\left(\begin{array}{l}j \\ k\end{array}\right)\left(\begin{array}{l}n-j \\ i-k\end{array}\right) \exp (\pi i s(2 k-i-j))$

for $0 \leq i, j \leq n$ 
We normalize a right invariant measure $d \mathbf{g}$ on $\mathbf{G}_{\mathbf{R}}^{+}$by $d((\xi, g))=|\operatorname{det} g|^{-2 m-2 n}$ $\Pi_{1 \leq i, j \leq n} d \operatorname{Re}\left(g_{i, j}\right) d \operatorname{Im}\left(g_{i, j}\right) \Pi_{i=1}^{m} \Pi_{j=1}^{n} d \operatorname{Re}\left(\xi_{i, j}\right) d \operatorname{Im}\left(\xi_{i, j}\right)$. Define the density $\mu(x)$ (resp. $\left.\mu^{*}\left(x^{*}\right)\right)$ for $\mathbf{x} \in \mathbf{V}_{\mathbf{Q}}-\mathbf{S}_{\mathbf{Q}}$ (resp. $\mathbf{x}^{*} \in \mathbf{V}_{\mathbf{Q}}-\mathbf{S}_{\mathbf{R}}^{*}$ ) as in $\S 6$ and set $\boldsymbol{\Gamma}=\left\{(\xi, \gamma) \in \mathbf{G}_{\mathbf{Q}} \mid\right.$ $\left.\xi \in \mathrm{M}_{m, n}\left(o_{K}\right), \gamma \in G L_{n}\left(o_{K}\right)\right\}$. Let $\mathbf{L}$ be a $\boldsymbol{\Gamma}$-admissible lattice of $\mathbf{V}_{\mathbf{Q}}$ with respect to $(\rho, \alpha)$ and $\mathbf{L}^{*}$ its dual. Put $\mathbf{L}_{i}=\mathbf{L} \cap \mathbf{V}_{i}$ and $\mathbf{L}_{i}^{*}=\mathbf{L}^{*} \cap \mathbf{V}_{i}^{*}(0 \leq i \leq n)$ and define zeta functions as follows:

$$
\begin{gathered}
\xi_{i}(s, \mathbf{L})=\sum_{[x, u] \in \mathbf{L}_{i} / \rho(\boldsymbol{\Gamma})} \mu([x, u]) \mathbf{e}\left[-\operatorname{tr}\left(x^{-1} H[u]\right)\right]|\operatorname{det} x|^{-s}, \\
\left.\xi_{i}^{*}\left(s, \mathbf{L}^{*}\right)=\sum_{[x, u] \in \mathbf{L}_{i}^{*} / \rho^{*}(\boldsymbol{\Gamma})} \mu^{*}([x, u]) \mid \operatorname{det}(x-H[u])\right]\left.\right|^{-s} .
\end{gathered}
$$

Then the condition (6.5) is satisfied. The following result is a consequence of Theorem 6.4 and Theorem 7.1 .

THEOREM 7.2. Let the notation be the same as in Theorem 7.1. The Dirichlet series $\xi_{i}(s, \mathbf{L})$ and $\xi_{i}^{*}\left(s, \mathbf{L}^{*}\right)$ are continued to meromorphic functions on $\mathbf{C}$ with possible simple poles at $s=1, \ldots, n$. Furthermore they satisfy the functional equations

$$
\begin{aligned}
& \operatorname{vol}\left(\mathbf{V}_{\mathbf{R}} / \mathbf{L}^{*}\right) \xi_{i}^{*}\left(m+n-s, \mathbf{L}^{*}\right) \\
& \quad=c(m+n-s) \gamma(s-m-n) \sum_{j=0}^{n} A_{j i}(s-m-n) \xi_{j}(s, \mathbf{L}) \quad(1 \leq i \leq n) .
\end{aligned}
$$

\section{REFERENCES}

[1] Brylinski, J-L. and Delorme, P., Vecteurs distributions $H$-invariants pour les séries principales généralisées d'espaces symetriques reductifs et prolongement meromorphe d'intégrales d'Eisenstein, Invent. Math., 109 (1992), 619-664.

[2] Murase, A. and Sugano, T., A note on zeta functions associated with certain prehomogeneous affine spaces, Advanced Studies in Pure Math., 15 (1989), 415-428.

[3] Sato, F., Zeta functions in several variables associated with prehomogeneous vector spaces I: Functional equations, Tohoku Math. J., 34 (1982), 437-483.

[4] Sato, F., On functional equations of zeta distributions, Advanced Studies in Pure Math., 15 (1989), 465-508.

[5] Sato, M., Theory of prehomogeneous vector spaces (notes by T. Shintani in Japanese), Sugaku no Ayumi, 15 (1970), 85-157.

[6] Sato, M., Kashiwara, M., Kimura, T., Oshima, T., Micro local analysis of prehomogeneous vector spaces, Invent. Math., 62 (1980), 117-179.

[7] Sato, M., Kimura, T., A classification of irreducible prehomogeneous vector spaces and their invariants, Nagoya Math. J., 65 (1977), 1-155.

[8] Sato, M., Shintani, T., On zeta functions associated with prehomogeneous vector spaces, Ann. of Math., 100 (1974), 131-170. 
Atsushi Murase

Faculty of Science

Kyoto Sangyo University

Motoyama Kamigamo

603 Kyoto, Japan

Takashi Sugano

Faculty of Science

Hiroshima University

1-3-1 Kagamiyama

724 Higashi-Hiroshima, Japan 